\title{
Evaluating the application and utility of ESSENCE for early event detection
}

\author{
Allison K. Kunerth*, Elizabeth Baker, Alan Zelicoff, Michael Elliot and Kevin Syberg
}

Saint Louis University, Saint Louis, MO, USA

\section{Objective}

A mixed methods study is being conducted on the statewide Early Notification of Community Based Epidemics (ESSENCE) system in Missouri to identify factors that can improve the timeliness and identification of outbreaks. This research will provide stakeholders with guidance on how best to implement and improve ESSENCE usage statewide, and by sharing this research input can be solicited on the utility of the applied framework as well as future implications from this body of work.

\section{Introduction}

In spite of the noted benefits of syndromic surveillance, and more than a decade after it started gaining support, the primary use for syndromic surveillance appears to be largely for seasonal and jurisdictional disease monitoring, event response and situational awareness as opposed to its intended purpose of early event detection. (1-4) Research assessing the user characteristics and standards applied at local public health agencies (LPHA's) for syndromic surveillance are scarce, and in national surveys epidemiologists frequently tend to utilize their own syndromic surveillance systems as opposed to a national system such as Biosense. While the National Syndromic Surveillance Program (NSSP) has addressed many operational concerns from stakeholders, and is in the process of providing access to the cloud based Biosense platform-along with ESSENCE as a key tool, there is still a paucity of research that exists as to what can be done to improve the utilization of syndromic surveillance systems for its primary purpose of early event detection.

\section{Methods}

This research proposes to evaluate the use of ESSENCE within Missouri and the surrounding areas, to comprehensively identify its strengths and limitations, through an assessment of the user experience. This research will evaluate three key areas: 1) the quality of the data received by the syndromic surveillance system, 2) the characteristics of the individuals and organizations utilizing the system (end-users), 3) the influence and extent of syndromic surveillance data on public health actions. ESSENCE data will be evaluated directly with special attention to the top three data quality attributes across the literature, completeness, accuracy and timeliness. (5) A survey will also be administered to ESSENCE system users and public health leadership at LPHA's, to gain insight into perspectives, perceptions and general practices, as well as how they interact with data from ESSENCE.

\section{Results}

The data for this research is primarily being collected throughout the fall of 2016, so the hope is to bring preliminary data to this conference as a means to validate some of the findings, solicit input on the proposed framework and share this research in a timely manner for the NSSP roll out of Biosense and ESSENCE.

\section{Conclusions}

Through a thorough evaluation, the application and utility of ESSENCE for early event detection will be better understood, along with the identification of factors that can be targeted in the future (and across syndromic surveillance platforms) for improvement in the timely identification of outbreaks.

Keywords

ESSENCE; syndromic surveillance; data quality

\section{Acknowledgments}

The data for this research is supplied by the Missouri Department of Health and Senior Services.

\section{References}

1. Chu A, Savage R, Willison D, Crowcroft NS, Rosella LC, Sider D, et al. The use of syndromic surveillance for decision-making during the H1N1 pandemic: A qualitative study. BMC Public Health. 2012;12(1):1-9. doi: 10.1186/1471-2458-12-929.

2. Samoff E, Fangman MT, Hakenewerth A, Ising A, Waller AE. Use of Syndromic Surveillance at Local Health Departments: Movement Toward More Effective Systems. Journal of Public Health Management and Practice. 2014;20(4):E25-E30. doi: 10.1097/ PHH.0b013e3182a505ac. PubMed PMID: WOS:000337137700003.

3. Buehler JW, Sonricker A, Paladini M, Soper P, Mostashari F. Syndromic surveillance practice in the United States: findings from a survey of state, territorial, and selected local health departments. Advances in Disease Surveillance. 2008;6(3):1-20.

4. Buehler JW, Whitney EA, Smith D, Prietula MJ, Stanton SH, Isakov AP. Situational uses of syndromic surveillance. Biosecurity and bioterrorism : biodefense strategy, practice, and science. 2009;7. doi: 10.1089/bsp.2009.0013.

5. Chen H, Hailey D, Wang N, Yu P. A review of data quality assessment methods for public health information systems. International journal of environmental research and public health. 2014;11(5):5170-207. doi: 10.3390/ijerph110505170. PubMed PMID: 24830450; PubMed Central PMCID: PMCPMC4053886.

\section{*Allison K. Kunerth}

E-mail: akunerth@slu.edu 